\title{
A meta-analysis of Hashimoto's thyroiditis and papillary thyroid carcinoma risk
}

\author{
Xingjian Lai ${ }^{1}$, Yu Xia ${ }^{1}$, Bo Zhang ${ }^{1}$, Jianchu $\mathrm{Li}^{1}$ and Yuxin Jiang ${ }^{1}$ \\ ${ }^{1}$ Department of Ultrasound, Chinese Academy of Medical Sciences \& Peking Union Medical College Hospital, Beijing, China \\ Correspondence to: Yu Xia, email: xiayupumch@163.com \\ Yuxin Jiang, email: jiangyxpumch@163.com \\ Keywords: Hashimoto's thyroiditis, chronic lymphocytic thyroiditis, papillary thyroid carcinoma, thyroid cancer, meta-analysis \\ Received: February 04, $2017 \quad$ Accepted: May 29, $2017 \quad$ Published: June 27, 2017 \\ Copyright: Lai et al. This is an open-access article distributed under the terms of the Creative Commons Attribution License 3.0 \\ (CC BY 3.0), which permits unrestricted use, distribution, and reproduction in any medium, provided the original author and source \\ are credited.
}

\section{ABSTRACT}

Objective: It remains inconclusive whether Hashimoto's thyroiditis (HT) predisposes patients to the development of papillary thyroid carcinoma (PTC). We conducted a meta-analysis of the available data to address this question.

Results: Twenty-seven eligible studies were selected, including 18 archival thyroidectomy studies, 6 fine-needle aspiration (FNA) studies, and 3 selective FNA or thyroidectomy studies. A total of 76,281 patients, including 12,476 cases of thyroid cancer, were included in these studies. The mean rate of PTC among patients with $\mathrm{HT}$ ranged from $1.12 \%$ (selective FNA or thyroidectomy studies) to $\mathbf{4 0 . 1 1 \%}$ (thyroidectomy studies). All three types of studies supported the correlation between HT and PTC. The overall pooled odds ratio (OR) of the PTC risk for HT (HT versus nonHT) was 2.12 (95\% confidence interval [CI]: 1.78-2.52).

Methods: We searched all relevant published studies using the citation databases PubMed and Embase. The ORs and corresponding 95\% CIs were calculated by the random-effects model for the association between HT and PTC.

Conclusions: Our meta-analysis confirmed that HT predisposed patients to the development of PTC.

\section{INTRODUCTION}

Hashimoto's thyroiditis (HT) (or chronic lymphocytic thyroiditis) is the most common autoimmune thyroid disease which causes the immune system to attack and destroy the thyroid gland [1]. Papillary thyroid carcinoma (PTC) is the most prevalent thyroid cancer and it represents $80-90 \%$ of all thyroid cancers. Dailey et al. were the first to propose an association between HT and PTC in 1955, linking chronic inflammation to neoplastic changes [2]. However, whether HT predisposes patients to the development of PTC is a continuing debate. Some studies have revealed a higher risk of PTC in patients with HT [3-20], while others did not demonstrate the increased risk [21-23]. Lee et al. published a metaanalysis and found that PTC is significantly associated with pathologically confirmed HT [24]. However,
Jankovic et al. found that there was no statistically significant correlation between HT and PTC based on 8 fine-needle aspiration (FNA) studies, and 8 thyroidectomy studies, which reported a statistically significant positive correlation, were subject to selection bias [25]. Because inconsistent results have been reported regarding the association between HT and PTC risk, and because many other related studies have been published in recent years, we performed a comprehensive meta-analysis to investigate the possible associations of HT and PTC.

\section{RESULTS}

\section{Study characteristics}

The literature search strategy identified 514 potentially eligible reports. Finally, 27 studies met the 
inclusion criteria, including 5 cohort studies $[6,11,16,21$, 26] and 22 case-control studies [3-5, 7-10, 12-15, 17-20, 22, 23, 27-32] (Figure 1). The detailed characteristics of these studies are shown in Table 1. Eleven studies were conducted in Europe, 9 in Asia, and 7 in the United States. Twenty-four studies evaluated the association between HT and PTC, and 3 studies evaluated the associations between HT and thyroid cancer. A total of 76,281 patients, including 12,476 cases of thyroid cancer, were included in these studies. The quality scores ranged from 5 to 8 with a median of 6 for methodological assessment.

Three types of studies were analyzed in this metaanalysis. The first type consists of studies of archival thyroidectomy specimens that were analyzed for the coexistence of HT and PTC. In the second type of study, all patients underwent FNA. However, only selected patients with results suspicious for malignancy underwent thyroidectomy. In the third type of study, only selected patients with suspicious thyroid nodules underwent FNA or thyroidectomy.

\section{Rate of PTC in patients with HT}

The rate of PTC in patients with HT from the 18 archival thyroidectomy studies (47,235 patients) ranged from $9.46 \%$ to $96.67 \%$, with a mean rate of $40.11 \%$ (Table 1, Figure 2). Two Korean studies revealed a significantly higher rate than the other thyroidectomy studies $(96.67 \%$ and $92.75 \%)[3,10]$. If those studies are excluded as possible outliers, the average rate in the archival thyroidectomy group would be $33.28 \%$.
The rate of PTC among patients with HT from the 6 FNA studies $(25,777$ patients) ranged from $0.12 \%$ to $20.87 \%$, with a mean rate of $6.46 \%$. Azizi et al. found a significantly higher rate than that in the other FNA studies $(20.87 \%)$ [4]. If that study is excluded as a possible outlier, the average prevalence rate in the FNA group would be $3.58 \%$.

The rate of PTC among patients with HT from the 3 selective FNA or thyroidectomy studies (11,793 patients) ranged from $0.67 \%$ to $1.77 \%$, with a mean rate of $1.12 \%$.

\section{OR of PTC in patients with HT}

Figure 3 shows study-specific data, including the odds ratio (OR) and corresponding 95\% confidence interval (CI) of the PTC risk for HT (HT versus nonHT). The pooled OR, based on overall studies, was 2.12 (95\% CI: 1.78-2.52). However, there was significant heterogeneity among the studies $\left(\mathrm{I}^{2}=84 \%, \mathrm{P}<0.00001\right)$. The corresponding estimates were 2.46 (95\% CI: $1.07-$ 5.66) for the cohort studies and 2.03 (95\% CI: 1.722.41) for the case-control studies. However, there were significant heterogeneities among the cohort studies $\left(\mathrm{I}^{2}=\right.$ $81 \%, \mathrm{P}=0.0003)$ and among the case-control studies $\left(\mathrm{I}^{2}=\right.$ $83 \%, \mathrm{P}<0.00001)$.

Figure 4 shows a specific study method and the OR and $95 \% \mathrm{CI}$ of thyroid cancer risk for HT (HT versus nonHT). The OR of PTC in patients with HT in the archival thyroidectomy group (18 studies) ranged from 1.34 to 4.40 , with an average OR of 2.34 for a total of 47,271 studied patients $\left(\mathrm{I}^{2}=81 \%, \mathrm{P}<0.00001\right)$. The OR of PTC

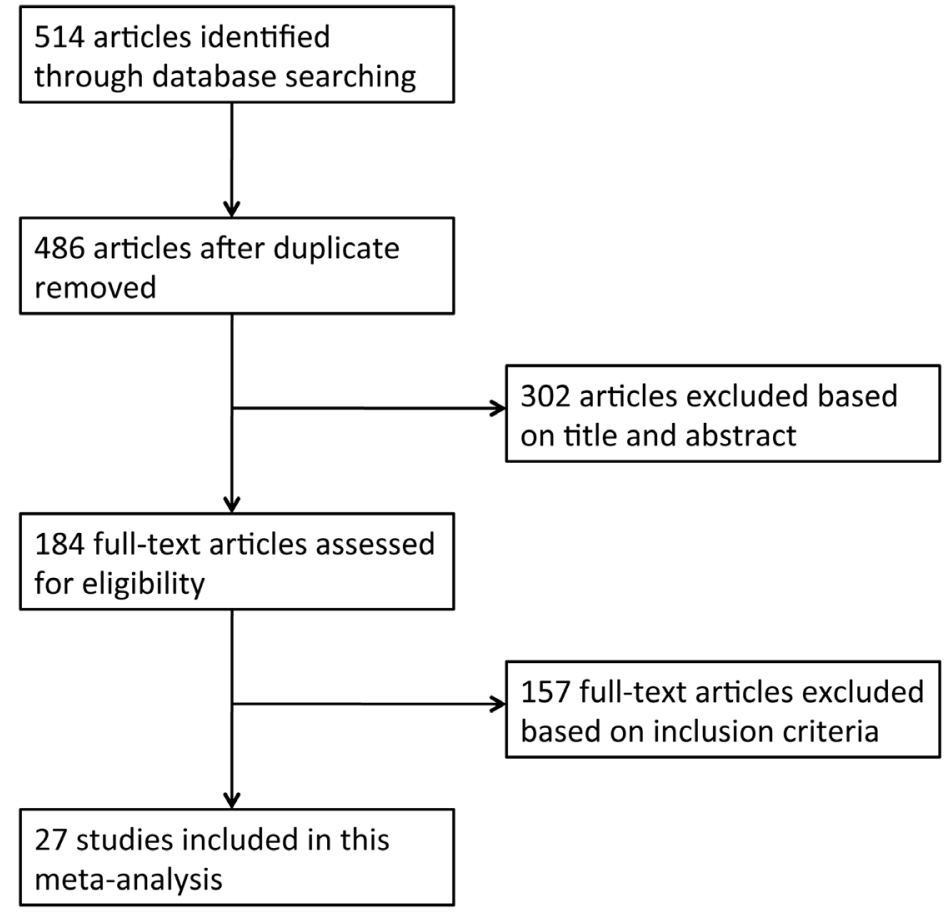

Figure 1: Flow diagram of article selection for this meta-analysis. 
Table 1: Characteristics of the studies included in the meta-analysis

\begin{tabular}{|c|c|c|c|c|c|c|c|c|}
\hline Study & Country & $\begin{array}{c}\text { Period of } \\
\text { enrolment }\end{array}$ & $\begin{array}{l}\text { Study } \\
\text { design }\end{array}$ & Study method & $\begin{array}{l}\text { Tumor } \\
\text { type }\end{array}$ & $\begin{array}{l}\text { Sample } \\
\text { size }\end{array}$ & $\begin{array}{l}\text { No. of } \\
\text { PTCs }\end{array}$ & $\begin{array}{l}\text { PTC in } \\
\text { HT, \% }\end{array}$ \\
\hline Chen 2013 [6] & Taiwan & $1998-2010$ & cohort & Selective FNA & $\mathrm{TC}$ & 7605 & 19 & 0.92 \\
\hline Isik 2010 [22] & Turkey & 2005-2009 & case-control & Selective FNA & $\mathrm{TC}$ & 500 & 5 & 0.67 \\
\hline Mukasa 2011 [16] & Japan & 2006 & cohort & Selective FNA & PTC & 3688 & 52 & 1.77 \\
\hline Anil 2010 [21] & Turkey & 2006-2009 & cohort & FNA & PTC & 715 & 21 & 1.22 \\
\hline Azizi 2014 [4] & USA & 2010-2013 & case-control & FNA & $\mathrm{TC}$ & 2023 & 233 & 20.87 \\
\hline $\begin{array}{l}\text { de Alcantara-Jones } \\
2015 \text { [28] }\end{array}$ & Brazil & 2011 & case-control & FNA & PTC & 1049 & 33 & 5.20 \\
\hline Fiore 2011 [8] & Italy & 2004-2009 & case-control & FNA & PTC & 9824 & 659 & 9.41 \\
\hline Holm 1985 [26] & Sweden & 1959-1978 & cohort & FNA & PTC & 1658 & 2 & 0.12 \\
\hline $\begin{array}{l}\text { Matesa-Anic } 2009 \\
{[23]}\end{array}$ & Croatia & 1995-2006 & case-control & FNA & PTC & 10508 & 269 & 1.95 \\
\hline Ahn 2011 [3] & Korea & $2000-2005$ & case-control & thyroidectomy & PTC & 303 & 269 & 96.67 \\
\hline Bradly 2009 [5] & USA & $2000-2008$ & case-control & thyroidectomy & PTC & 678 & 81 & 28.38 \\
\hline Buyukasik 2011 [27] & Turkey & 1999-2006 & case-control & thyroidectomy & PTC & 917 & 61 & 11.69 \\
\hline Consorti 2010 [7] & Italy & 1995-2008 & case-control & thyroidectomy & PTC & 404 & 101 & 36.23 \\
\hline Gul 2010 [9] & Turkey & $2005-2008$ & case-control & thyroidectomy & PTC & 613 & 193 & 45.65 \\
\hline Kim 2011 [10] & Korea & 2003-2007 & case-control & thyroidectomy & PTC & 1277 & 1028 & 92.75 \\
\hline Konturek 2013 [11] & Poland & $2002-2010$ & cohort & thyroidectomy & PTC & 7545 & 636 & 23.45 \\
\hline $\begin{array}{l}\text { Kurukahvecioglu } \\
2007 \text { [12] }\end{array}$ & Turkey & 2001-2005 & case-control & thyroidectomy & PTC & 922 & 199 & 36.63 \\
\hline Larson 2007 [13] & USA & 1987-2002 & case-control & thyroidectomy & PTC & 812 & 179 & 34.69 \\
\hline Liu 2014 [14] & China & $2008-2013$ & case-control & thyroidectomy & PTC & 6432 & 1722 & 43.75 \\
\hline Lun 2013 [15] & China & 2004-2012 & case-control & thyroidectomy & PTC & 2478 & 676 & 49.61 \\
\hline $\begin{array}{l}\text { Mazokopakis } 2010 \\
\text { [29] }\end{array}$ & Greece & 2005-2009 & case-control & thyroidectomy & PTC & 140 & 32 & 28.57 \\
\hline McLeod 1988 [30] & USA & 1975-1985 & case-control & thyroidectomy & PTC & 793 & 143 & 23.44 \\
\hline Paparodis 2014 [17] & USA & 1994-2013 & case-control & thyroidectomy & PTC & 2733 & 893 & 44.16 \\
\hline Repplinger 2008 [31] & USA & 1994-2007 & case-control & thyroidectomy & PTC & 1198 & 293 & 29.03 \\
\hline Siriweera 2010 [18] & Sri Lanka & $\begin{array}{c}\text { Not } \\
\text { specified }\end{array}$ & case-control & thyroidectomy & PTC & 5357 & 145 & 9.46 \\
\hline Zhang 2012 [19] & China & $2008-2010$ & case-control & thyroidectomy & PTC & 6109 & 2797 & 58.35 \\
\hline Zhang 2014 [20] & China & 2004-2011 & case-control & thyroidectomy & PTC & 8524 & 1735 & 29.44 \\
\hline
\end{tabular}

No., number; PTC, papillary thyroid carcinoma; HT, Hashimoto’s thyroiditis; FNA, fine-needle aspiration; TC, thyroid cancer. 


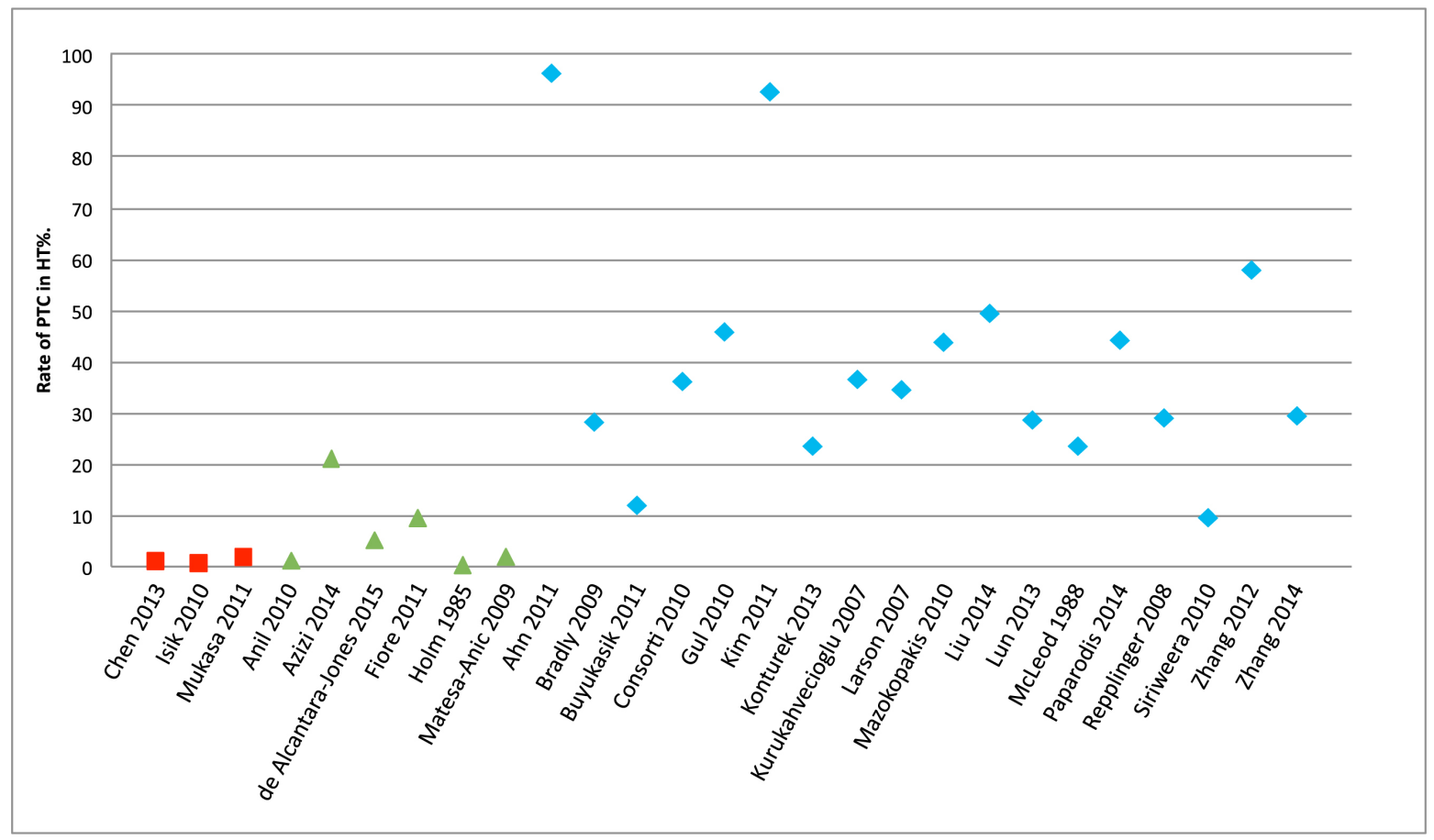

Figure 2: Rate of PTC among patients with HT. HT, Hashimoto's thyroiditis; PTC, papillary thyroid carcinoma. Note: The red squares indicate selective fine-needle aspiration or thyroidectomy studies, the green triangles indicate fine-needle aspiration studies, and the blue rhombuses indicate thyroidectomy studies.

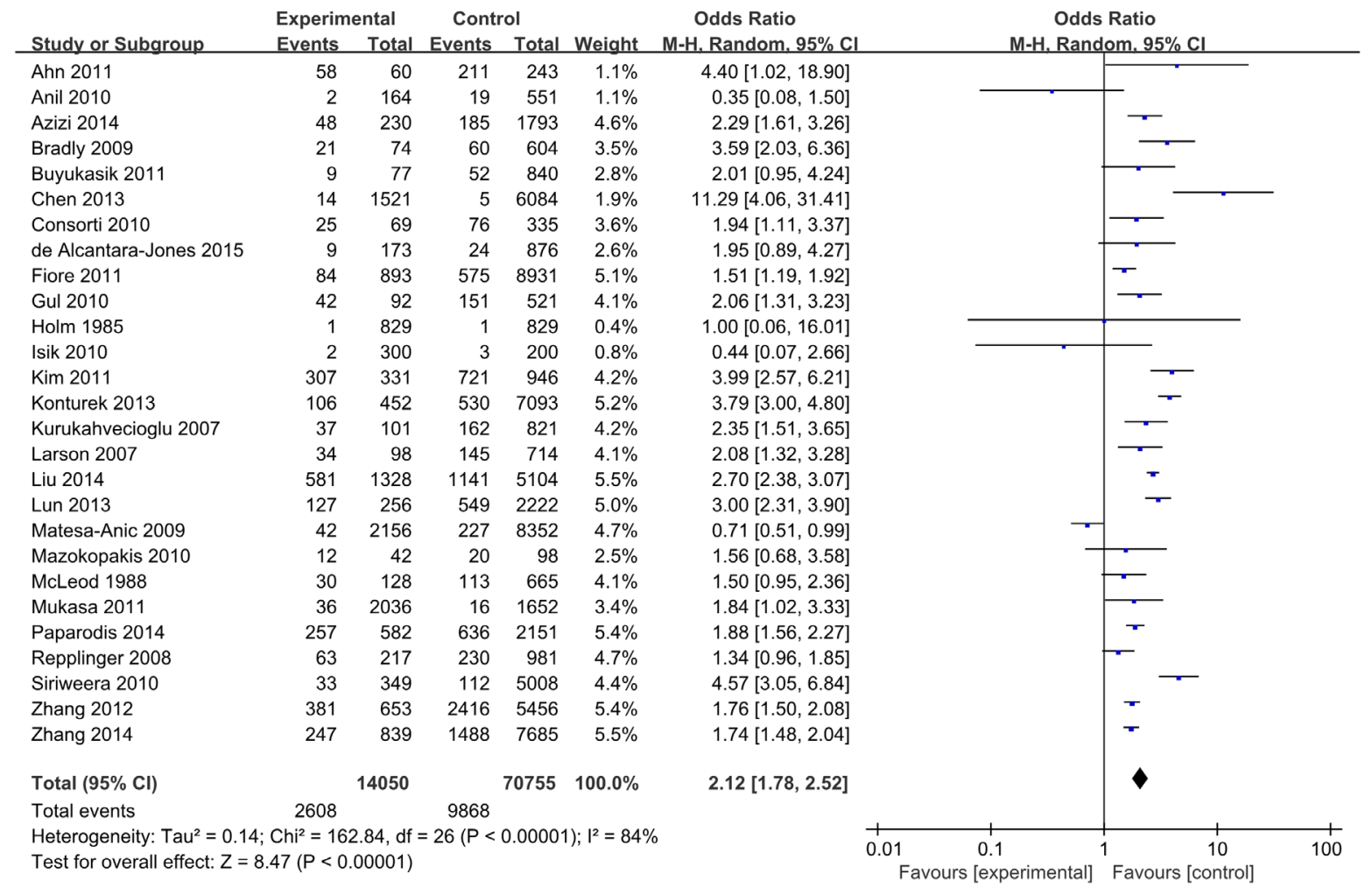

Figure 3: Pooled risk estimates of HT for PTC risk (HT versus non-HT). HT, Hashimoto's thyroiditis; PTC, papillary thyroid carcinoma. 
in patients with HT in the FNA group (6 studies) ranged from 0.35 to 2.29 , with an average OR of 1.27 for a total of 25,777 studied patients $\left(\mathrm{I}^{2}=82 \%, \mathrm{P}<0.0001\right)$. The OR of PTC in patients with HT in the selective FNA group (3 studies) ranged from 0.44 to 11.29 , with an average OR of 2.37 for a total of 11,793 studied patients $\left(\mathrm{I}^{2}=85 \%, \mathrm{P}\right.$ $=0.002$ ).

Table 2 presents the results of subgroup analyses regarding the association between HT and PTC risk. Considering the geographical area, the pooled OR for PTC risk was 1.56 (95\% CI: 1.01-2.40) in European studies, 2.79 (95\% CI: 2.15-3.61) in Asian studies, and 1.92 (95\% CI: 1.56-2.35) in American studies. However, significant heterogeneity was found within the geographic areas (all P-values were $<0.10)$.

\section{Sensitivity analysis and publication bias}

The sensitivity analyses revealed that no study had a significant influence on the overall estimates. The pooled ORs for HT varied from 2.04 (when excluding Siriweera et al. [18]) to 2.24 (when excluding Matesa-Anic et al. [23]). The shape of the funnel plot of studies assessing the association between HT and PTC risk seemed to be symmetrical, indicating the absence of publication bias (Figure 5).

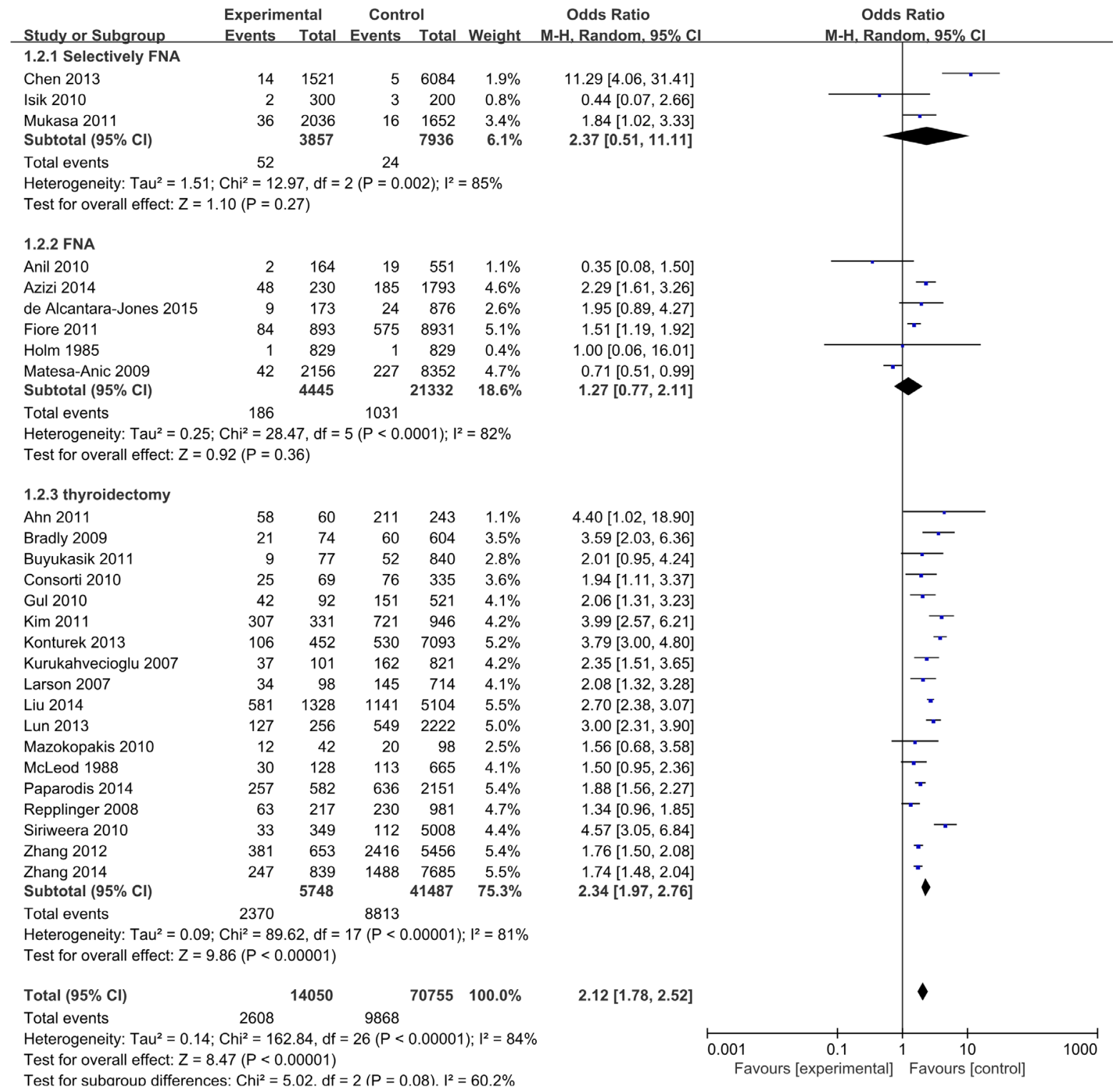

Figure 4: Pooled risk estimates of HT for PTC risk (HT versus non-HT) according to the strata of study method. HT, Hashimoto's thyroiditis; PTC, papillary thyroid carcinoma; FNA, fine needle aspiration. 
Table 2: Pooled risk estimates of HT for PTC risk (HT versus non-HT) according to the strata of selected covariates

\begin{tabular}{|c|c|c|c|}
\hline & No. of studies & OR $(95 \%$ CI $)$ & P for heterogeneity \\
\hline Overall & 27 & $2.12(1.78-2.52)$ & \\
\hline \multicolumn{4}{|l|}{ Study design } \\
\hline Cohort & 5 & $2.46(1.07-5.66)$ & 0.0003 \\
\hline Case-control & 22 & $2.03(1.72-2.41)$ & $<0.00001$ \\
\hline \multicolumn{4}{|l|}{ Study method } \\
\hline Thyroidectomy & 18 & $2.34(1.97-2.76)$ & $<0.00001$ \\
\hline FNA & 6 & $1.27(0.77-2.11)$ & $<0.0001$ \\
\hline Selective FNA & 3 & $2.37(0.51-11.11)$ & 0.002 \\
\hline \multicolumn{4}{|l|}{ Geographic area } \\
\hline Europe & 11 & $1.56(1.01-2.40)$ & $<0.00001$ \\
\hline Asia & 9 & $2.79(2.15-3.61)$ & $<0.00001$ \\
\hline America & 7 & $1.92(1.56-2.35)$ & 0.07 \\
\hline \multicolumn{4}{|l|}{ Publication year } \\
\hline$\geq 2010$ & 20 & $2.32(1.94-2.78)$ & $<0.00001$ \\
\hline$<2010$ & 7 & $1.65(1.06-2.55)$ & $<0.00001$ \\
\hline \multicolumn{4}{|l|}{ No. of PTCs } \\
\hline$\geq 100$ & 18 & $2.12(1.75-2.56)$ & $<0.00001$ \\
\hline$<100$ & 9 & $1.98(1.16-3.37)$ & 0.003 \\
\hline \multicolumn{4}{|l|}{ Tumor subtype } \\
\hline PTC & 24 & $2.06(1.73-2.47)$ & $<0.00001$ \\
\hline $\mathrm{TC}$ & 3 & $2.64(0.66-10.50)$ & 0.002 \\
\hline \multicolumn{4}{|l|}{ Quality score } \\
\hline$\geq 7$ & 2 & $4.79(0.49-46.92)$ & 0.11 \\
\hline$<7$ & 22 & $2.06(1.73-2.44)$ & $<0.00001$ \\
\hline \multicolumn{4}{|c|}{ Adjusted for multivariate } \\
\hline Yes & 6 & $2.33(1.32-4.14)$ & $<0.00001$ \\
\hline No & 21 & $1.88(1.51-2.34)$ & $<0.00001$ \\
\hline \multicolumn{4}{|l|}{ Mean age (year) } \\
\hline$\geq 48$ & 15 & $1.98(1.54-2.53)$ & $<0.00001$ \\
\hline$<48$ & 7 & $1.94(1.41-2.67)$ & 0.004 \\
\hline \multicolumn{4}{|c|}{ Sex ratio (female:male) } \\
\hline$\geq 5$ & 10 & $2.17(1.41-3.35)$ & $<0.00001$ \\
\hline$<5$ & 12 & $1.99(1.61-2.45)$ & $<0.00001$ \\
\hline \multicolumn{4}{|c|}{ Mean nodule size $(\mathrm{cm})$} \\
\hline$\geq 1.3$ & 5 & $2.09(1.64-2.66)$ & 0.005 \\
\hline$<1.3$ & 4 & $3.38(2.56-4.46)$ & 0.16 \\
\hline
\end{tabular}

No., number; OR, odds ratio; CI, confidence interval; FNA, fine-needle aspiration; PTC, papillary thyroid carcinoma; TC, thyroid cancer. 


\section{DISCUSSION}

This meta-analysis showed that PTC was more often found in patients with HT than in patients without HT. However, there was significant heterogeneity among the studies, and the potential explanations may include variations in study methods, different patient inclusion criteria, and heterogeneous diagnostic criteria for HT and PTC. A previous meta-analysis found similar heterogeneity $(\mathrm{Q}=39.664, \mathrm{df}=10, \mathrm{P}<0.001)$ [24].

In this meta-analysis, 3 types of studies were analyzed, namely, 3 selective FNA studies, 6 FNA studies, and 18 thyroidectomy studies. In the selective FNA studies, only selected patients underwent FNA or thyroidectomy; therefore, the rate of PTC among patients with HT $(1.12 \%)$ was lower than that in the FNA studies (3.58\%) and thyroidectomy studies (33.28\%). However, the OR of PTC risk for HT (HT versus non-HT) (2.37) was not lower than that in the FNA studies (1.27) and thyroidectomy studies (2.34).
In the 3 selective FNA studies, one study reported a negative association between HT and thyroid carcinoma. Isik et al. compared the prevalence and sonographic features of thyroid carcinoma in 300 HT and 200 Graves' disease (GD) patients [22]. In their study, all patients underwent ultrasonography, but only $32 \mathrm{HT}$ and 55 GD patients underwent total thyroidectomy. No patient underwent FNA. The indications for surgery included patient preference, compressive symptoms, severe ophthalmopathy, patients whose ultrasonography revealed features that needed investigation, large goiter, and failure and/or serious side effects of antithyroid drugs, including agranulocytosis and hepatotoxicity. Two and three cases of thyroid carcinoma were confirmed in HT and GD patients, respectively, and the OR of thyroid carcinoma risk was 0.44 (95\% CI: 0.07-2.66). In comparison, there were 19 and 52 cases of thyroid carcinoma in two other selective FNA studies $[6,16]$. Mukasa et al. also compared the prevalence of thyroid carcinoma in 2,036 HT and 1,652 GD patients [16]. They performed ultrasound-guided

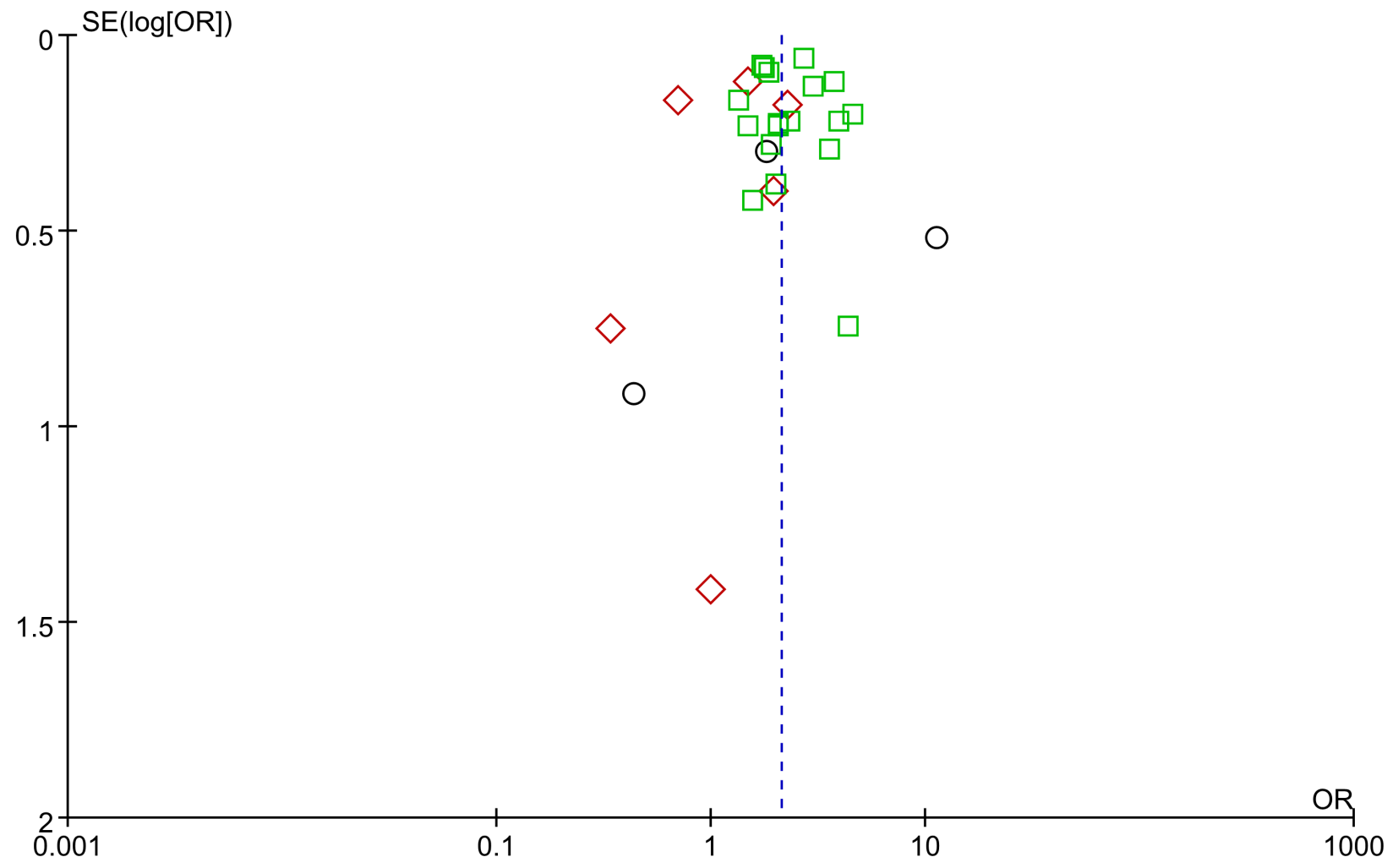

$\square$ Selectively FNA $\diamond_{\text {FNA }} \quad \square$ thyroidectomy

Figure 5: Funnel plot for publication bias in studies comparing the rate of PTC between HT and non-HT. PTC, papillary thyroid carcinoma; HT, Hashimoto's thyroiditis; FNA, fine-needle aspiration. Note: The black circles indicate selective FNA studies, the red rhombuses indicate FNA studies, and the green squares indicate thyroidectomy studies. 
FNA biopsy when the diameter of a nodule was $>1 \mathrm{~cm}$ or when a nodule was suspected of being malignant base on the ultrasonographic features, including the presence of microcalcifications, nodule hypoechogenicity, irregular margins, and a shape taller than wide. Fifty-two patients who underwent surgery due to a suspicion of PTC were diagnosed with PTC. The study conducted by Chen et al. used claims data from the Taiwan National Health Insurance Program, and each patient was examined from the index date to the occurrence of cancer, death, or withdrawal from insurance or until the end of 2010 [6]. Therefore, there were significant differences in the patient population and surgery indication in these selective FNA studies, providing one possible explanation for the significant heterogeneity $\left(\mathrm{I}^{2}=85 \%, \mathrm{P}=0.002\right)$.

There were also significant differences in the patient population of FNA studies. The inclusion criteria of FNA studies included consecutive patients $[23,28]$, patients with benign thyroid diseases [26], patients with cold thyroid nodules [8], patients with nodules $\geq 10 \mathrm{~mm}$ [21], and patients with nodules $\geq 5 \mathrm{~mm}$ and aged $\geq 18$ years [4]. This may be one explanation for the wide incidence range of PTC, from $0.12 \%$ to $20.87 \%$. Anil et al. prospectively compared the prevalence of thyroid carcinoma between 164 patients with HT and 551 patients without HT and found that thyroid nodules in patients with HT were no more likely to be malignant than those in patients without HT $(\mathrm{OR}=0.35,95 \%$ CI: 0.08-1.50). However, they included only patients with nodules $\geq 10 \mathrm{~mm}$. In addition, only $4.9 \%$ of their HT group and $10 \%$ of the control group underwent thyroidectomy for thyroid nodules [21].

All 18 thyroidectomy studies in this meta-analysis showed that PTC is more often found in patients with HT than in patients without HT. In the clinical review conducted by Jankovic et al., all 8 thyroidectomy studies showed similar results [25]. However, Jankovic et al. believed that selection bias led to the statistically significant positive correlation. Lee et al. found that HT was more frequently observed in PTCs than in benign thyroid diseases (OR: $2.8 ; \mathrm{P}<0.001)$. In the funnel plots and Egger's regression tests, there was no evidence of publication bias [24].

The relationship between inflammation and PTC is complex and still not completely understood [33]. Tamimi et al. found a significantly higher rate of lymphocytic infiltrate in patients with PTC, and the activated inflammatory response present in HT may create a favorable setting for malignant transformation [34]. The inflammatory response may cause DNA damage through the formation of reactive oxygen species, resulting in mutations that eventually lead to the development of PTC [25]. Both retrospective studies and a prospective study suggest that the association of HT with PTC is antibody-specific $[4,35,36]$. However, the mechanism underlying this association is not known. Paradoxically, the lymphocytic infiltrate of HT may be an immunological response with a cancer-retarding effect, contributing to a favorable outcome of PTC [37-39]. The relatively high prevalence of PTC in autopsy series may represent host immune control [5].

In conclusion, this meta-analysis includes the most comprehensive existing data on HT and PTC risk to date, with a large sample size, confirming that HT predisposed patients to the development of PTC. To better understand the potential mechanisms underlying this association, further studies are needed.

\section{MATERIALS AND METHODS}

\section{Search strategy and inclusion criteria}

We identified all relevant case-control and cohort studies published in English by searching two databases (PubMed and Embase) from the beginning of indexing to December 2015, using the following terms: (Hashimoto disease OR Hashimoto struma OR Hashimoto thyroiditis OR Hashimoto syndrome OR chronic lymphocytic thyroiditis) AND (thyroid tumor OR thyroid cancer OR thyroid carcinoma OR thyroid neoplasm) AND (cohort OR prospective OR case-control), following the Metaanalysis Of Observational Studies in Epidemiology (MOOSE) guidelines [40]. Two authors (XJ Lai and Y $\mathrm{Xia}$ ) independently assessed and identified potentially relevant articles and reviewed the reference lists in the articles and associated reviews to identify additional studies. The inclusion criteria were as follows: (1) cohort study or case-control study published as an original article; (2) evaluation of the association of HT and thyroid cancer; and (3) availability ofthe OR and corresponding 95\% CIs or sufficient information to enable their calculation. Abstracts or unpublished reports were not considered for inclusion in the meta-analysis.

\section{Data extraction and quality assessment}

Two authors (XJ Lai and Y Xia) independently extracted the data from each original article. The extracted data included the first author, year of publication, country, study design, study method, period of enrollment, sample size (numbers of cases, controls, or non-cases or cohort size), and OR for HT and corresponding 95\% CIs. Two authors (XJ Lai and Y Xia) independently assessed the quality of the included studies according to the NewcastleOttawa Scale (NOS) [41]. The NOS includes three broad perspectives: selection (four items), comparability (two items), and exposure/outcome (three items). The full score was 9 points, and a study with $\geq 7$ points was defined as a high-quality study. Disagreements were discussed and resolved through consensus. 


\section{Statistical analysis}

The ORs were used for the meta-analyses. The pooled ORs with $95 \%$ CIs were calculated by the randomeffects models for the association between HT and PTC [42]. Heterogeneity among articles was quantitatively assessed using the $\mathrm{Q}$ test and $\mathrm{I}^{2}$ statistic [43]. A significant heterogeneity was defined as $\mathrm{I}^{2}>50 \%$ or Q-test reporting a $\mathrm{P}$ value $<0.1$. To explore the potential sources of heterogeneity among studies, we conducted subgroup analyses for the strata of the study design, study method, geographic area, publication year, number of cases, tumor subtype, NOS quality score, whether adjusted for multivariate, mean age, sex ratio and mean nodule size. Sensitivity analyses were also performed by excluding one study at a time to clarify the influence of each study on the overall estimates. Publication bias was assessed by the funnel plot [44].

A P value $<0.05$ was considered to be statistically significant. All P values were two-tailed. Statistical analyses were performed using RevMan 5 (www. cochrane.org) or the SPSS 11.5 software package (SPSS, Chicago, IL).

\section{Abbreviations}

HT, Hashimoto's thyroiditis; PTC, papillary thyroid carcinoma; FNA, fine needle aspiration; OR, odds ratio; CI, confidence interval; GD, Graves' disease; NOS, Newcastle-Ottawa Scale.

\section{Author contributions}

Conceived and designed the meta-analysis: Bo Zhang, Yuxin Jiang. Performed the meta-analysis: Xingjian Lai, Yu Xia. Analyzed the data: Xingjian Lai, Yu Xia. Contributed reagents/materials/analysis tools: Jianchu Li, Yuxin Jiang. Wrote the paper: Xingjian Lai, Yu Xia.

\section{ACKNOWLEDGMENTS}

This work was supported by the Science and Technology Project of the Ministry of Labor and Personnel of China (M426300), and International Science and Technology Corporation Program of China (2015DFA30440).

\section{CONFLICTS OF INTEREST}

The authors declare no conflicts of interest.

\section{REFERENCES}

1. Dayan CM, Daniels GH. Chronic autoimmune thyroiditis. N Engl J Med. 1996; 2:99-107.
2. Dailey ME, Lindsay S, Skahen R. Relation of thyroid neoplasms to Hashimoto disease of the thyroid gland. AMA Arch Surg. 1955; 2:291-7.

3. Ahn D, Heo SJ, Park JH, Kim JH, Sohn JH, Park JY, Park SK, Park J. Clinical relationship between Hashimoto's thyroiditis and papillary thyroid cancer. Acta Oncol. 2011; 8:1228-34.

4. Azizi G, Keller JM, Lewis M, Piper K, Puett D, Rivenbark KM, Malchoff CD. Association of Hashimoto's thyroiditis with thyroid cancer. Endocr Relat Cancer. 2014; 6:845-52.

5. Bradly DP, Reddy V, Prinz RA, Gattuso P. Incidental papillary carcinoma in patients treated surgically for benign thyroid diseases. Surgery. 2009; 6:1099-104.

6. Chen YK, Lin CL, Cheng FT, Sung FC, Kao CH. Cancer risk in patients with Hashimoto's thyroiditis: a nationwide cohort study. Br J Cancer. 2013; 9:2496-501.

7. Consorti F, Loponte M, Milazzo F, Potasso L, Antonaci A. Risk of malignancy from thyroid nodular disease as an element of clinical management of patients with Hashimoto's thyroiditis. Eur Surg Res. 2010; 3-4:333-7.

8. Fiore E, Rago T, Latrofa F, Provenzale MA, Piaggi P, Delitala A, Scutari M, Basolo F, Di Coscio G, Grasso L, Pinchera A, Vitti P. Hashimoto's thyroiditis is associated with papillary thyroid carcinoma: role of TSH and of treatment with L-thyroxine. Endocr Relat Cancer. 2011; 4:429-37.

9. Gul K, Dirikoc A, Kiyak G, Ersoy PE, Ugras NS, Ersoy $\mathrm{R}$, Cakir B. The association between thyroid carcinoma and Hashimoto's thyroiditis: the ultrasonographic and histopathologic characteristics of malignant nodules. Thyroid. 2010; 8:873-8.

10. Kim KW, Park YJ, Kim EH, Park SY, Park DJ, Ahn SH, Park DJ, Jang HC, Cho BY. Elevated risk of papillary thyroid cancer in Korean patients with Hashimoto's thyroiditis. Head Neck. 2011; 5:691-5.

11. Konturek A, Barczynski M, Wierzchowski W, Stopa M, Nowak W. Coexistence of papillary thyroid cancer with Hashimoto thyroiditis. Langenbecks Arch Surg. 2013; 3:389-94.

12. Kurukahvecioglu O, Taneri F, Yuksel O, Aydin A, Tezel E, Onuk E. Total thyroidectomy for the treatment of Hashimoto's thyroiditis coexisting with papillary thyroid carcinoma. Adv Ther. 2007; 3:510-6.

13. Larson SD, Jackson LN, Riall TS, Uchida T, Thomas RP, Qiu S, Evers BM. Increased incidence of well-differentiated thyroid cancer associated with Hashimoto thyroiditis and the role of the PI3k/Akt pathway. J Am Coll Surg. 2007; 5:764-73, discussion 773-5.

14. Liu X, Zhu L, Cui D, Wang Z, Chen H, Duan Y, Shen M, Wu Y, Rong R, Zhang Z, Wang X, Chen J, Alexander $\mathrm{EK}$, et al. Coexistence of histologically confirmed Hashimoto's thyroiditis with different stages of papillary thyroid carcinoma in a consecutive Chinese cohort. Int $\mathbf{J}$ Endocrinol. 2014; 2014:769294. 
15. Lun Y, Wu X, Xia Q, Han Y, Zhang X, Liu Z, Wang F, Duan Z, Xin S, Zhang J. Hashimoto's thyroiditis as a risk factor of papillary thyroid cancer may improve cancer prognosis. Otolaryngol Head Neck Surg. 2013; 3:396-402.

16. Mukasa K, Noh JY, Kunii Y, Matsumoto M, Sato S, Yasuda S, Suzuki M, Ito K, Ito K. Prevalence of malignant tumors and adenomatous lesions detected by ultrasonographic screening in patients with autoimmune thyroid diseases. Thyroid. 2011; 1:37-41.

17. Paparodis R, Imam S, Todorova-Koteva K, Staii A, Jaume JC. Hashimoto's thyroiditis pathology and risk for thyroid cancer. Thyroid. 2014; 7:1107-14.

18. Siriweera EH, Ratnatunga NV. Profile of Hashimoto's thyroiditis in Sri Lankans: is there an increased risk of ancillary pathologies in Hashimoto's thyroiditis? J Thyroid Res. 2010; 2010:124264.

19. Zhang L, Li H, Ji QH, Zhu YX, Wang ZY, Wang Y, Huang CP, Shen Q, Li DS, Wu Y. The clinical features of papillary thyroid cancer in Hashimoto's thyroiditis patients from an area with a high prevalence of Hashimoto's disease. BMC Cancer. 2012; 12:610.

20. Zhang Y, Dai J, Wu T, Yang N, Yin Z. The study of the coexistence of Hashimoto's thyroiditis with papillary thyroid carcinoma. J Cancer Res Clin Oncol. 2014; 6:1021-6.

21. Anil C, Goksel S, Gursoy A. Hashimoto's thyroiditis is not associated with increased risk of thyroid cancer in patients with thyroid nodules: a single-center prospective study. Thyroid. 2010; 6:601-6.

22. Isik S, Gokay F, Ozuguz U, Topaloglu O, Tutuncu Y, Berker $\mathrm{D}$, Guler S. Comparison of the prevalence and sonographic features of thyroid nodules accompanying autoimmune thyroid diseases. Endokrynol Pol. 2010; 6:658-64.

23. Matesa-Anic D, Matesa N, Dabelic N, Kusic Z. Coexistence of papillary carcinoma and Hashimoto's thyroiditis. Acta Clin Croat. 2009; 1:9-12.

24. Lee JH, Kim Y, Choi JW, Kim YS. The association between papillary thyroid carcinoma and histologically proven Hashimoto's thyroiditis: a meta-analysis. Eur J Endocrinol. 2013; 3:343-9.

25. Jankovic B, Le KT, Hershman JM. Hashimoto's thyroiditis and papillary thyroid carcinoma: is there a correlation? $\mathrm{J}$ Clin Endocrinol Metab. 2013; 2:474-82.

26. Holm LE, Blomgren H, Lowhagen T. Cancer risks in patients with chronic lymphocytic thyroiditis. N Engl J Med. 1985; 10:601-4.

27. Buyukasik O, Hasdemir AO, Yalcin E, Celep B, Sengul S, Yandakci K, Tunc G, Kucukpinar T, Alkoy S, Col C. The association between thyroid malignancy and chronic lymphocytic thyroiditis: should it alter the surgical approach? Endokrynol Pol. 2011; 4:303-8.

28. de Alcantara-Jones DM, de Alcantara-Nunes TF, Rocha BO, de Oliveira RD, Santana AC, de Alcantara FT, de Faria TM, Da SI, Araujo LM. Is there any association between
Hashimoto's thyroiditis and thyroid cancer? A retrospective data analysis. Radiol Bras. 2015; 3:148-53.

29. Mazokopakis EE, Tzortzinis AA, Dalieraki-Ott EI, Tsartsalis AN, Syros PK, Karefilakis CM, Papadomanolaki MG, Starakis IK. Coexistence of Hashimoto's thyroiditis with papillary thyroid carcinoma. A retrospective study. Hormones (Athens). 2010; 4:312-7.

30. McLeod MK, East ME, Burney RE, Harness JK, Thompson NW. Hashimoto's thyroiditis revisited: the association with thyroid cancer remains obscure. World J Surg. 1988; 4:509-16.

31. Repplinger D, Bargren A, Zhang YW, Adler JT, Haymart M, Chen H. Is Hashimoto's thyroiditis a risk factor for papillary thyroid cancer? J Surg Res. 2008; 1:49-52.

32. Singh B, Shaha AR, Trivedi H, Carew JF, Poluri A, Shah JP. Coexistent Hashimoto's thyroiditis with papillary thyroid carcinoma: impact on presentation, management, and outcome. Surgery. 1999; 6:1070-6, discussion 1076-7.

33. Guarino V, Castellone MD, Avilla E, Melillo RM. Thyroid cancer and inflammation. Mol Cell Endocrinol. 2010; 1:94-102.

34. Tamimi DM. The association between chronic lymphocytic thyroiditis and thyroid tumors. Int J Surg Pathol. 2002; 2:141-6.

35. Azizi G, Malchoff CD. Autoimmune thyroid disease: a risk factor for thyroid cancer. Endocr Pract. 2011; 2:201-9.

36. Kim ES, Lim DJ, Baek KH, Lee JM, Kim MK, Kwon HS, Song KH, Kang MI, Cha BY, Lee KW, Son HY. Thyroglobulin antibody is associated with increased cancer risk in thyroid nodules. Thyroid. 2010; 8:885-91.

37. Huang BY, Hseuh C, Chao TC, Lin KJ, Lin JD. Welldifferentiated thyroid carcinoma with concomitant Hashimoto's thyroiditis present with less aggressive clinical stage and low recurrence. Endocr Pathol. 2011; 3:144-9.

38. Kim EY, Kim WG, Kim WB, Kim TY, Kim JM, Ryu JS, Hong SJ, Gong G, Shong YK. Coexistence of chronic lymphocytic thyroiditis is associated with lower recurrence rates in patients with papillary thyroid carcinoma. Clin Endocrinol (Oxf). 2009; 4:581-6.

39. Matsubayashi S, Kawai K, Matsumoto Y, Mukuta T, Morita T, Hirai K, Matsuzuka F, Kakudoh K, Kuma K, Tamai $\mathrm{H}$. The correlation between papillary thyroid carcinoma and lymphocytic infiltration in the thyroid gland. J Clin Endocrinol Metab. 1995; 12:3421-4.

40. Stroup DF, Berlin JA, Morton SC, Olkin I, Williamson GD, Rennie D, Moher D, Becker BJ, Sipe TA, Thacker SB. Meta-analysis of observational studies in epidemiology: a proposal for reporting. Meta-analysis Of Observational Studies in Epidemiology (MOOSE) group. JAMA. 2000; 15:2008-12.

41. Stang A. Critical evaluation of the Newcastle-Ottawa scale for the assessment of the quality of nonrandomized studies in meta-analyses. Eur J Epidemiol. 2010; 9:603-5. 
42. DerSimonian R, Laird N. Meta-analysis in clinical trials. Control Clin Trials. 1986; 3:177-88.

43. Higgins JP, Thompson SG. Quantifying heterogeneity in a meta-analysis. Stat Med. 2002; 11:1539-58.
44. Peters JL, Sutton AJ, Jones DR, Abrams KR, Rushton L. Contour-enhanced meta-analysis funnel plots help distinguish publication bias from other causes of asymmetry. J Clin Epidemiol. 2008; 10:991-6. 